\title{
The Role of Procalcitonin as a Prognostic Variable in Ovarian Cancer Patients at Dr. Soetomo General Hospital Surabaya
}

\author{
Irma Savitri, Brahmana Askandar Tjokroprawiro*
}

Division of Gynecologic Oncology, Department of Obstetrics and Gynecology, Dr. Soetomo General Academic Hospital, Medical Faculty - Universitas Airlangga, Surabaya, Indonesia

\section{ARTICLE INFO}

Received : 06 February 2020

Reviewed : 02 November 2020

Accepted : 22 April 2021

\section{Keywords:}

ovarian tumor, procalcitonin level, stage, resectability

\author{
*Corresponding author: \\ Brahmana Askandar Tjokroprawiro \\ Division of Gynecologic Oncology, \\ Department of Obstetrics and \\ Gynecology, Dr. Soetomo General \\ Academic Hospital, Medical Faculty \\ - Universitas Airlangga, Surabaya, \\ Indonesia \\ brahmanaaskandar@fk.unair.ac.id
}

\begin{abstract}
A BSTRACT
Background: Procalcitonin (PCT) is a pro-hormone calcitonin that is strongly associated with bacterial infections, especially bacteremia. High serum PCT levels have been correlated with Lung cancer, colorectal cancer, thyroid cancer, and tumors of neuroendocrine. Studies on the role of PCT in ovarian tumors are still limited. This study aims to evaluate the role of PCT as a prognostic variable to determine the effectiveness of diagnostics in predicting ovarian cancer progression and metastasis.
\end{abstract}

Methods: This was a descriptive analytic study with a cross sectional design, conducted in Dr. Soetomo General Hospital Surabaya from July until October 2019. The subject selection used total quota sampling with inclusion criteria of female aged $>12$ or already menstruating, undergoing surgical removal of ovarian tumors either cystic or solid, with risk malignancy index (RMI) of $>200$, and with imaging results of suggestive ovarian malignancy. The data were analyzed descriptively using Chi-square test $(\alpha<0.05)$ and receiver operating characteristic (ROC) curve analysis.

Results: There were 36 study subjects; $38.9 \%$ of them are $40-49$ years old; $77.8 \%$ have malignant tumors; and $22.2 \%$ of them have benign tumors with epithelial histopathology of $78.5 \%$ and nonepithelial of $21.4 \%$. The optimal cut-off for PCT levels in ovarian tumors is $0.07 \mathrm{ng} / \mathrm{ml}$ with a $57.1 \%$ sensitivity and $87.5 \%$ specificity. There was a significant correlation between levels of PCT and ovarian tumors based on tumor types, postoperative tumor residues, and stages $(p<0.05)$. There was no significant correlation between PCT levels and histopathological types and tumor grade.

Conclusions: Procalcitonin can be used as a prognostic variable to determine disease progression in ovarian tumors, especially in ovarian cancer.

\section{INTRODUCTION}

Ovarian cancer is the most common cause of gynecological cancer-related deaths among women in developed countries. Several studies to identify the prognostic factors that characterize ovarian cancer patients including tumor characteristics, surgical management, genetic patterns, immunological factors, and genome patterns are underway. Continued efforts to identify these factors can result in valuable additional information for the treatment of ovarian cancer with the ultimate goal of developing the management of patients with ovarian cancer [1].
There are difficulties in early detection, diagnosis, treatment, and overall survival (OS) rate of patients with malignant ovarian cancer. Epithelial ovarian cancer (EOC) is the deadliest gynecological cancer. Some factors that can affect OS include stage, histological type, early detection, patient management, age, and comorbidity [2]. The staging of ovarian cancer and the presence of postoperative tumor residual mass in primary cytoreductive surgery are of the most importance in predicting the outcomes in patients with ovarian cancer. Definitions of optimal surgical cytoreduction following 
completion of the initial surgery have varied in the literature from no macroscopic residual disease to 3 $\mathrm{cm}$ gross residual disease. In the United States, the Gynecology Oncology Group (GOG) has settled on $1 \mathrm{~cm}$ or less residual disease as a criterion for optimum cytoreductive surgery [3].

Several systems have been developed to assess the resectability and metastatic extent of ovarian cancer. One of them is the peritoneal cancer index $(\mathrm{PCl})$. The use of $\mathrm{PCl}$ in ovarian cancer has been widely studied with results where $\mathrm{PCl}$ is the scoring system that best predicts resectability in addition to the modified Fagotti score. $\mathrm{PCl}$ is reliable and easy to do with the same results by different operators and may be used as a prognostic indicator of resectability in ovarian cancer. Peritoneal cancer index assessment was performed intraoperatively [4].

Several previous studies have shown elevated levels of serum PCT in patients with cancer, especially with neuroendocrine origin and hepatocellular carcinoma. Many previous studies have evaluated the role of PCT in lung cancer, colorectal cancer, and thyroid cancer. In fact, a study found positive levels of PCT in the serum of patients with different malignancies (thyroid adenocarcinoma, testicular tumors, prostate cancer, melanoma, ovarian cancer, Ewing sarcoma, gastrointestinal and pancreatic cancer, and breast cancer) [3]. A study by Keramidaris et al. [5] which examined the relationship between PCT levels and colorectal cancer stated that their study showed that PCT was significantly higher in patients with metastasis and marginally significant with tumor grade. Matzaraki et al. [6] showed that patients with solid tumors, metastases, and no evidence of infection had markedly elevated PCT levels, especially those with generalized metastatic disease. Our study finds out whether high PCT levels can be used as a prognostic variable to predict the stages in ovarian cancer and postoperative tumor residues because until now, there has not been a single biomarker that can predict the stages in ovarian cancer or determine the possibility of postoperative tumor residues. As we know, tumor stage and postoperative residual tumor mass at primary cytoreductive surgery have been shown to most reliably predict outcome in patients with ovarian cancer [7].

The aim of this study is to evaluate the role of PCT as a prognostic variable to determine the effectiveness of diagnostics in predicting cancer progression and metastasis. Progressive at least a $20 \%$ increase in the sum of the longest diameter of the target lesion, taking as reference is the smallest summation of the longest diameter recorded since treatment began or the appearance of one or more new lesions. Metastasis is the spread of disease or cancer cells from one organ to another either directly or indirectly. Our hypothesis is that the increased PCT levels preoperative in ovarian cancer patients is associated with high staging and the presence of distant metastases in ovarian cancer, which is also associated with an increased risk of cytoreductive surgery with a residue of $>1 \mathrm{~cm}$ in the primary surgery. Increased serum PCT levels is useful for selecting patients in which it is more beneficial to be give neoadjuvant chemotherapy therapy to ovarian cancer patients compared with primary cytoreductive surgery.

\section{METHODS}

The study was conducted in Dr. Soetomo General Hospital Surabaya at the Gynecology Oncology outpatient Department, inpatient, and operating room from July until October 2019. Laboratory tests were carried out at the Clinical and Anatomical Pathology Laboratory, Faculty of Medicine, Airlangga University. The design of this study is descriptive analytic with a cross sectional design. The selection of research subjects was carried out in total quota sampling. Inclusion criteria includes female patients aged $\geq 12$ or already menstruating, willing for surgical removal of ovarian tumors, having risk malignancy index (RMI) $>200$ in patients with suspected malignancy, and imaging that supports malignancy, and patients with either cystic or solid ovarian tumors. Residual tumors are the remaining tumors with a size of more than or equal to $1 \mathrm{~cm}$ with a very tight attachment to the intra-abdominal organs such as the small intestine, large intestine, peritoneum, liver, and/or the presence of a milliary tumor.

The data collected were coded and entered into Statistical Package for Social Sciences (SPSS) version 21. Frequency and percentages were used in descriptive measurement with Chi-square test $(\alpha<0.05)$ and receiver operating characteristic (ROC) curve for data analysis. Then, the optimal cut-off point for the test was determined based on its ROC curve. Lastly, the associated sensitivity and specificity were estimated.

\section{RESULTS}

During the study period, there were 40 subjects, four of which were dropped out. Two subjects were dropped out because the tumor originated not from the ovary, 1 due to poor general condition that we have to wait for the improvement in general condition first, and the other due to lung metastasis and respiratory failure until the patient finally died. This study was followed by 36 subjects who met the inclusion criteria, and the exclusion criteria were not obtained. The optimal cut-off for PCT levels in this study was $0.07 \mathrm{ng} / \mathrm{ml}$ (Figure 1). The cut-off point for PCT levels used in the study is based on the AUC value (area under the curve) on the AUC graph from the results of statistical calculations. 


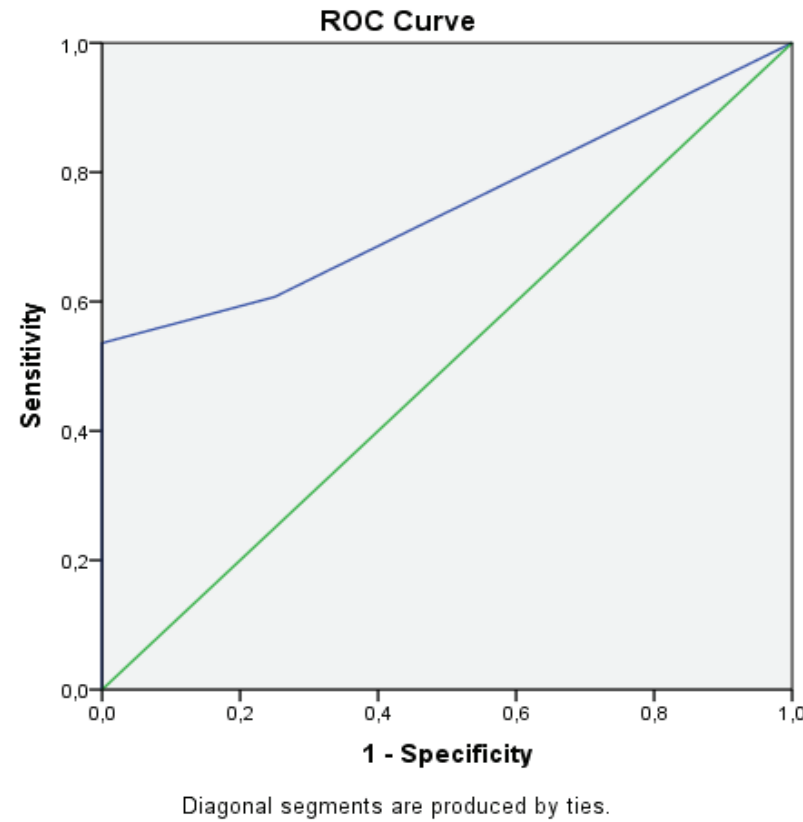

Figure 1. ROC curve for procalcitonin

Table 1. Tabulation table of procalcitonin levels associated with tumor characteristics.

\begin{tabular}{|c|c|c|c|c|c|}
\hline \multirow{2}{*}{\multicolumn{2}{|c|}{ Characteristics }} & \multirow{3}{*}{$\begin{array}{l}\mathbf{N} \\
28\end{array}$} & \multicolumn{2}{|c|}{ Procalcitonin Level } & \multirow[b]{2}{*}{$\mathbf{p}$} \\
\hline & & & \multirow{2}{*}{$\begin{array}{l}\geq 0.07 \\
\mathbf{N}(\%) \\
16(57.1)\end{array}$} & \multirow{2}{*}{$\begin{array}{l}<0.07 \\
\mathbf{N}(\%) \\
12(42.9)\end{array}$} & \\
\hline Types & Malignant & & & & $0.044^{*}$ \\
\hline & Benign & 8 & $1(12.5)$ & $7(87.5)$ & \\
\hline \multirow[t]{2}{*}{ Residue } & Positive & 11 & $9(81.8)$ & $2(18.2)$ & $0.017^{*}$ \\
\hline & Negative & 25 & $8(32.0)$ & $17(68.0)$ & \\
\hline \multirow[t]{4}{*}{ Stages } & 1 & 12 & $3(25.0)$ & $9(75.0)$ & $0.024 *$ \\
\hline & 2 & 4 & $3(75.0)$ & $1(25.0)$ & \\
\hline & 3 & 9 & 7 (77.7) & $2(22.2)$ & \\
\hline & 4 & 3 & $3(100.0)$ & $0(0.0)$ & \\
\hline \multirow{2}{*}{$\begin{array}{l}\text { Types } \\
\text { of Histo- } \\
\text { pathology }\end{array}$} & Epithelial & 22 & $14(63.6)$ & $8(36.4)$ & 0.354 \\
\hline & $\begin{array}{l}\text { Non- } \\
\text { Epithelial }\end{array}$ & 6 & $2(33.3)$ & $4(66.6)$ & \\
\hline \multirow[t]{2}{*}{ Grade } & Low & 11 & $6(54.5)$ & $5(45.5)$ & 0.659 \\
\hline & High & 11 & $8(72.7)$ & $3(27.3)$ & \\
\hline
\end{tabular}

*statistically significant $(p<0.05)$

Table 1 shows that PCT levels are associated with types of malignant or benign tumors. Procalcitonin levels are significantly associated with types of tumors, tumor residues, and staging with $p=0.044, p=0.017$, and $p=0.024$. There is no significant correlation between PCT levels with histopathological types and ovarian cancer grading with $p=0.354$ and $p=0.659$.

\section{DISCUSSION}

Our study at Dr. Soetomo General Hospital was conducted to see whether PCT levels which have been used as infection markers have a role as a prognostic variable in patients with ovarian tumor at Dr. Soetomo General Hospital. So far, there is only one other study by Hefler et al. [7] that examined the C-reactive protein in ovarian cancer where this inflammatory biomarker acts as a prognostic factor in ovarian cancer.

On established prognostic factors, postoperative residual tumor mass and ovarian tumor stage have been shown to be the most reliable predictors of outcome in ovarian cancer [8]. The high morbidity of patients with ovarian cancer undergoing primary cytoreductive surgery with advanced stages and many residues makes the author want to find a biomarker tool that can reduce that risk. Procalcitonin as a biomarker of infection is thought to determine the progression of the disease and the metastases of ovarian cancer. In some types of solid tumor malignancies such as medullary thyroid cancer and small cell lung cancer, several studies have proven the effectiveness of procalcitonin to detect malignancies and progression of the disease. The predicting factor studied before was peritoneal cancer index $(\mathrm{PCl}) . \mathrm{PCl}$ is the scoring system that best predicts resectability in addition to the modified Fagotti score, but unfortunately this scoring can only be done to assess the resectability and prognosis of ovarian cancer at intraoperative time, not preoperatively [7].

From the results of our study, PCT levels examined preoperatively in each sample compared with benign and malignant tumors in the ovaries were significantly correlated. This suggests that PCT has a role in predicting ovarian cancer as it appears that PCT levels are higher in ovarian cancer than in benign ovarian tumors. Based on the stage in ovarian cancer, there is a significant correlation. Procalcitonin levels in ovarian cancer are higher in ovarian cancer in advanced stage compared to ovarian cancer in early stage. Patients with distant metastases have PCT levels higher than patients without distant metastases. This shows that PCT has a role in assessing the progression of ovarian cancer. This is consistent with the study conducted by Chaftari et al. [3] on cancers with solid tumors, suggesting that PCT levels in cancer patients with metastases are significantly higher in cancer patients without metastase. This is also supported by the study conducted by Matzaraki et al. [5] in which PCT levels increase in cancer patients who have metastasized, specifically in a group of patients with total metastases. Procalcitonin levels in the blood can be used as an initial indicator for the progression of neoplasmic disease regardless of the specificity of the disease metastases. 
The terms cytoreductive surgery and debulking are used interchangeably to indicate surgical efforts aimed at removing most of the tumors. Complete cytoreduction is achieved when no tumor is seen after surgery. The Gynecologic Oncology Group currently defines optimal cytoreduction as having tumor residues of $1 \mathrm{~cm}$ or less in maximum diameter, each, or as no residual tumors. No tumor residue has proven to produce better overall survival than suboptimal cytoreduction and be a better predictor of survival [6].

In our study, PCT levels are significantly associated with postoperative tumor residues. The tumor residues found in this study were in several places including the bladder, liver, colon, and rectosigmoid area, and most of them were residues on milliary tumors in the colon, omentum, and peritoneum areas. The ultimate goal of surgery is to achieve complete resection without visible or palpable residual disease in the abdomen. In our study, there was a significant correlation between procalcitonin levels and postoperative tumor residues. This is also supported by a study conducted by Matzaraki et al. [6] where the results of his research said that PCT levels increased in cancer patients who had metastasized specifically in the group of patients with complete metastatic disease. PCT levels in the blood can be used as an early indicator for the progression of neoplastic disease regardless of the specificity of the spread of the disease. Based on the stage of ovarian cancer, there is a significant correlation between procalcitonin levels compared to the stage of ovarian cancer. Procalcitonin levels in ovarian cancer are higher in ovarian cancer with advanced stage than in early stage ovarian cancer. Patients with distant metastases had higher procalcitonin levels than patients without distant metastases. This suggests that procalcitonin has a role in assessing the progression of ovarian cancer. This is in accordance with a study conducted by Chaftary et al. [3] who conducted a study on cancer with solid tumors which showed that PCT levels in cancer patients with overall spread were significantly higher in cancer patients without metastases.

The results of this study indicate that PCT levels in the blood can function as clinically useful markers in patients with ovarian cancer where elevated serum PCT levels may predict tumor residues after primary cytoreductive surgery. This could be useful for the selection of patients who benefit from neoadjuvant chemotherapy compared to those who benefit from primary cytoreductive surgery.

Based on the type of histopathology between epithelial and non-epithelial ovarian cancers compared with PCT levels, they are not significantly correlated. The grade of the tumor is not significantly correlated either.

\section{CONCLUSIONS}

Preoperative procalcitonin levels are increased in patients with ovarian cancer compared with benign ovarian tumor patients. Procalcitonin levels are increased in patients with advanced-stage ovarian cancer with distant metastases and postoperative tumor residues greater than $1 \mathrm{~cm}$. In this study, procalcitonin levels is not associated with histopathological type and tumor grade.

\section{DECLARATIONS}

\section{Competing of Interest}

I declare that I do not have any competing interests.

\section{Ethics Approval}

Ethical approval was obtained from the hospital ethics committee of Dr. Soetomo General Hospital Surabaya with the registration number 1251/KEPK/VI/2019.

\section{Acknowledgment}

The author would like to thank the entire academic community at Dr. Soetomo General Hospital for giving the opportunity to conduct this study and the Department of Obstetrics and Gynecology, Faculty of Medicine, Airlangga University. Thanks to the Division of Gynecology Oncology who fully support the implementation of this study from the beginning to completion. Not to forget, the author expresses her gratitude to the Department of Obstetrics and Gynecology of Faculty of Medicine, Hasanuddin University as the institution of origin.

\section{REFERENCES}

1. Ezzati M, Abdullah A, Shariftabrizi A, et al. Recent advancements in prognostic factors of epithelial ovarian carcinoma. Int Sch Res Notices. 2014;2014:953509.

2. Chang L, Huang C, Lai M, et al. Prognostic factors in epithelial ovarian cancer: A population-based study. PLoS One. 2018;13(3):e0194993.

3. Chaftari AM, Hachem R, Reitzel R, et al. Role of procalcitonin and interleukin- 6 in predicting cancer, and its progression independent of infection. PLoS One. 2015;10(7):e0130999.

4. Harmon RL, Sugarbaker PH. Prognostic indicators in peritoneal carcinomatosis from gastrointestinal cancer. Int Semin Surg Oncol. 2005;2(1):3.

5. Keramidaris D, Koronakis N, Lagoudianakis EE, et al. Procalcitonin in patients with colorectal cancer. J BUON. 2013;18(3):623-8 
6. Matzaraki V, Alexandraki KI, Venetsanou K, et al. Evaluation of serum procalcitonin and interleukin- 6 levels as markers of liver metastasis. Clin Biochem. 2007;40(5-6):336-42.

7. Hefler LA, Concin N, Hofstetter G, et al. Serum C-reactive protein as independent prognostic variable in patients with ovarian cancer. Clin Cancer Res. 2008;14(3):710-4.

8. Liu $Y$, Chen $S$, Zheng $C$, et al. The prognostic value of the preoperative c-reactive protein/albumin ratio in ovarian cancer. BMC Cancer. 2017;17(1):285. 